\title{
On the structure of Banach spaces with an unconditional basic sequence
}

\author{
by \\ Razvan Anisca (Thunder Bay)
}

\begin{abstract}
For a Banach space $X$ with an unconditional basic sequence, one of the following regular-irregular alternatives holds: either $X$ contains a subspace isomorphic to $\ell_{2}$, or $X$ contains a subspace which has an unconditional finite-dimensional decomposition, but does not admit such a decomposition with a uniform bound for the dimensions of the decomposition. This result can be viewed in the context of Gowers' dichotomy theorem.
\end{abstract}

1. Introduction. Following the general constructions of Komorowski and Tomczak-Jaegermann [9]-[11], [14] of subspaces without an unconditional basis for a large class of Banach spaces, in this paper we continue the investigation of properties related to unconditionality in general Banach spaces.

Our main result provides the following alternative regarding the structure of Banach spaces with an unconditional basic sequence:

THeOREM 1.1. Let $X$ be a Banach space which has an infinite-dimensional subspace $Y$ with an unconditional basis. Then one of the following holds:

(i) $Y$ contains a subspace isomorphic to $\ell_{2}$,

(ii) $Y$ contains a subspace which has an unconditional finite-dimensional decomposition, but does not admit such a decomposition with a uniform bound for the dimensions of the decomposition.

We can also see this result from the point of view of Gowers' dichotomy theorem [6]: every infinite-dimensional Banach space has an infinite-dimensional subspace which is either hereditarily indecomposable or has an unconditional basis. Theorem 1.1 provides some further information in the second case, in the form of an additional "regular-irregular" alternative.

2000 Mathematics Subject Classification: Primary 46B20; Secondary 46B15.

Key words and phrases: unconditional basis, unconditional decomposition. 
The proof is done by working in the same setting as Komorowski and Tomczak-Jaegermann and using their construction recursively; in the process we exhibit, for every $n \geq 2$, a subspace $Y_{n}$ which admits an unconditional decomposition into $n$-dimensional subspaces and for which $n$ is minimal with such property. These subspaces (which are described in Sections 3 and 4) constitute the main ingredients used in deducing Theorem 1.1 (in Section 5). Constructions with similar properties were previously obtained for subspaces of $L_{p}(1 \leq p<2)$ by Borzyszkowski [2], and for subspaces of $\ell_{2}(X)$ when $X$ is a non-hilbertian Banach space with finite cotype ([1]).

The constructions of this paper relate also to the problem regarding the number of non-isomorphic infinite-dimensional subspaces of a Banach space which is not isomorphic to $\ell_{2}$. It was shown recently by Ferenczi and Rosendal [4] that any hereditarily indecomposable Banach space has a continuum of mutually non-isomorphic infinite-dimensional subspaces, while for spaces with an unconditional basic sequence the same is true under certain assumptions (see [4, Theorem 8 and Corollary 14]). If one tries to relax those assumptions and requires that the space (with an unconditional basic sequence) is not $\ell_{2}$-saturated, then the family of subspaces $\left\{Y_{n}\right\}_{n}$ provides a countable family of non-isomorphic subspaces.

2. Notation and preliminaries. We start with a certain amount of standard notation from the Banach space theory. We refer the reader to [12], [13] for all notation not explained here.

For $C \geq 1$, a basis $\left\{e_{i}\right\}_{i}$ in a Banach space $X$ is called $C$-unconditional if for every $x=\sum_{i} a_{i} e_{i} \in X$ one has $\left\|\sum_{i} \varepsilon_{i} a_{i} e_{i}\right\| \leq C\|x\|$ for all signs $\varepsilon_{i}= \pm 1$. If $\left\{e_{i}\right\}_{i}$ is an unconditional basis in $X$ then there exists an equivalent norm on $X$ under which $\left\{e_{i}\right\}_{i}$ is 1-unconditional.

A Banach space is said to have a finite-dimensional decomposition $\left\{Z_{k}\right\}_{k}$ if every vector $x \in X$ has a unique representation $x=\sum_{k} z_{k}$ such that $z_{k} \in Z_{k}$ and $\operatorname{dim} Z_{k}<\infty$ for all $k \geq 1$. We refer to such a decomposition $\left\{Z_{k}\right\}_{k}$ as uniform if $\sup _{k} \operatorname{dim} Z_{k}<\infty$ and as s-uniform if $\sup _{k} \operatorname{dim} Z_{k} \leq s$. A decomposition $\left\{Z_{k}\right\}_{k}$ is called $C$-unconditional, for some $C \geq 1$, if for all $x=\sum_{k} z_{k} \in X$ as above and all signs $\varepsilon_{k}= \pm 1$, one has $\left\|\sum_{k} \varepsilon_{k} z_{k}\right\| \leq C\|x\|$.

The following definition ([2]) represents a generalization of the classical notion of local unconditional structure.

Definition 2.1. A Banach space $X$ has local unconditional structure of order $\leq s$ if there is $C \geq 1$ such that for every finite-dimensional subspace $E \subset X$ there exist a Banach space $V$ and operators $u: E \rightarrow V, w: V \rightarrow X$ such that $i_{E}=w u, V$ has a 1-unconditional s-uniform decomposition and $\|u\|\|w\| \leq C$. Here $i_{E}$ is the natural embedding $i_{E}: E \rightarrow X$. The infimum of such constants $C$ is denoted by $\mathcal{U}_{s}(X)$. 
While it is clear that, for a Banach space $X$, the property $\mathcal{U}_{s}(X)<\infty$ is weaker than $X$ having an $s$-uniform decomposition, this notion has the advantage of passing to complemented subspaces: if $P: X \rightarrow X$ is a bounded projection then $\mathcal{U}_{s}(P X) \leq\|P\| \mathcal{U}_{s}(X)$.

The following result, due to Ketonen [8] and Borzyszkowski [2], is our fundamental criterion for recognizing that a space does not have local unconditional structure of order $\leq s$. It has the roots in the approach first introduced by Johnson, Lindenstrauss and Schechtman [7] for investigating the Kalton-Peck space.

Proposition 2.2. Let $Z$ be a Banach space of cotype $r$ for some $r<\infty$. Assume that $Z$ has a $C$-unconditional $k$-uniform decomposition $\left\{Z_{i}\right\}_{i}$ for some $k \geq 2$ and $C \geq 1$. Suppose also that there exists $s \leq k-1$ such that $\mathcal{U}_{s}(Z)<\infty$. Then there is a bounded linear operator operator $T: Z \rightarrow Z$ such that

(i) $T\left(Z_{i}\right) \subset Z_{i}$ for $i=1,2, \ldots$,

(ii) $\left\|T_{\mid Z_{i}}-\theta I_{Z_{i}}\right\| \geq 1 /\left(2 k^{2}\right)$ for each $\theta \in \mathbb{R}$ and $i=1,2, \ldots$

It should be mentioned that more information is known about the operator $T$ above: in particular, an estimate for $\|T\|$ in terms of the parameters involved (cotype $r$ constant, $\mathcal{U}_{s}(Z)$ and $C$ ) is obtained by Komorowski and Tomczak-Jaegermann in [9], [10] (see also [1]); as well, $T$ can be chosen such that $T_{\mid Z_{i}}$ is a non-trivial projection for every $i=1,2, \ldots$, as was shown by Casazza and Kalton [3] (see also [1]).

We now describe the general setting in which Komorowski and TomczakJaegermann constructed spaces without unconditional basis. This setting consists of a direct sum of several Banach spaces with unconditional bases and the construction relies on the behavior of subsequences of these bases with respect to certain partitions of $\mathbb{N}$. In order to be more precise we need the following notations. If $\Delta=\left\{A_{m}\right\}_{m}$ and $\Delta^{\prime}=\left\{A_{m}^{\prime}\right\}_{m}$ are two partitions of $\mathbb{N}$, we say that $\Delta \succ \Delta^{\prime}$ if the partition $\Delta^{\prime}$ is obtained by taking successive members of $\Delta$ : there are $1=k_{1}<\cdots<k_{m}<\cdots$ such that

$$
A_{m}^{\prime}=\bigcup_{j=k_{m}}^{k_{m+1}-1} A_{j} \quad \text { for } m=1,2, \ldots
$$

In such a situation, for $m=1,2, \ldots$, we set

$$
\mathcal{K}\left(A_{m}^{\prime}, \Delta\right)=\left\{K \subset A_{m}^{\prime}:\left|K \cap A_{j}\right|=1 \text { for } k_{m} \leq j<k_{m+1}\right\} .
$$

If $\Delta_{i}=\left\{A_{i, m}\right\}_{m}$ is a sequence of partitions $(i=1,2, \ldots)$ with $\Delta_{1} \succ$ $\ldots \succ \Delta_{i} \succ \ldots$, we let, for $m=1,2, \ldots$ and $i=2,3, \ldots$,

$$
\mathcal{K}_{i, m}=\mathcal{K}\left(A_{i, m}, \Delta_{i-1}\right) .
$$


Let $Y$ be a Banach space with a 1-unconditional basis $\left\{e_{l}\right\}_{l}$ and suppose that $Y$ does not contain a subspace isomorphic to $\ell_{2}$. Let $N$ be a positive integer. It was proved in [9] (see also [14]), using spreading models and Krivine's theorem, that one can always find a 1-unconditional decomposition $F_{1} \oplus \cdots \oplus F_{N} \subset Y$ with some special properties. Namely, for each $i=1, \ldots, N$ the space $F_{i}$ has a normalized 1-unconditional basis $\left\{f_{i, l}\right\}_{l}$ and there exist partitions $\Delta_{1} \succ \cdots \succ \Delta_{N}$ of $\mathbb{N}, \Delta_{i}=\left\{A_{i, m}\right\}_{m}$, such that for $i=1, \ldots, N-1$ and $m=1,2, \ldots$ and for all scalars $\left\{a_{k}\right\}_{k}$ we have

$$
\left\|\sum_{k \in A_{i, m}} a_{k} f_{i+1, k}\right\| \leq 4\left\|\sum_{k \in A_{i, m}} a_{k} f_{i, k}\right\|
$$

and one of the following conditions is satisfied:

(I) for every $i=1, \ldots, N-1$ and $m=1,2, \ldots$ and every set $K \in \mathcal{K}_{i+1, m}$ there exists a sequence $\left\{\beta_{k}\right\}_{k \in K}$ of scalars such that

$$
\left\|\sum_{k \in K} \beta_{k}\left(f_{1, k}+\cdots+f_{i, k}\right)\right\|=1, \quad\left\|\sum_{k \in K} \beta_{k} f_{i+1, k}\right\| \geq 2^{m} .
$$

(II) for every $i=1, \ldots, N-1$ and $m=1,2, \ldots$ and every set $K \in \mathcal{K}_{i+1, m}$ there exists a sequence $\left\{\beta_{k}\right\}_{k \in K}$ of scalars such that

$$
\left\|\sum_{k \in K} \beta_{k} f_{i+1, k}\right\|=1, \quad\left\|\sum_{k \in K} \beta_{k} f_{i, k}\right\| \geq 2^{m} .
$$

REMARK 2.3. The subspaces $F_{1}, \ldots, F_{N} \subset Y$ are supported on disjoint subsets of the unconditional basis $\left\{e_{l}\right\}_{l}$ of $Y$. Furthermore, for each $i=$ $1, \ldots, N$, the basis $\left\{f_{i, l}\right\}_{l}$ is obtained by taking suitable disjoint blocks of $\left\{e_{l}\right\}_{l}$, except for the situation when the vectors $\left\{e_{l}\right\}_{l}$ span the space $\ell_{p}$ (for some $1 \leq p<\infty, p \neq 2)$, in which case the construction is a consequence of the fact that $Y \simeq\left(\sum_{n} \oplus \ell_{2}^{n}\right)_{\ell_{p}}$. Then $F_{1} \oplus \cdots \oplus F_{N}=\left(F_{1} \oplus \cdots \oplus F_{N}\right)_{\ell_{p}}$.

For our constructions we use the same setting and thus we have to split the argument into two parts, according to which one of the alternatives (I) or (II) holds true. In Section 3 we treat case (I), while in Section 4, which follows the same line of argument, we deal with case (II).

3. Recursive construction in case (I). Let $Y$ be a Banach space with a 1-unconditional basis. Suppose that $Y$ does not contain a subspace isomorphic to $\ell_{2}$ and that it has finite cotype. For the remainder of the section we will assume that for every $N \geq 1$ we can find 1-unconditional decompositions $F_{1} \oplus \cdots \oplus F_{N} \subset Y$ satisfying the conditions (2) and (3).

Let $n \geq 2$. Our main goal in this section is to construct a subspace $Y_{n} \subset Y$ which admits a 1-unconditional decomposition $Y_{n}=\overline{\operatorname{span}}\left\{Z_{k}\right\}_{k \geq 1}$ with $\operatorname{dim} Z_{k}=n$ for all $k \geq 1$ and, at the same time, $\mathcal{U}_{n-1}\left(Y_{n}\right)=\infty$. 
More precisely, given an arbitrary function $g: \mathbb{N} \rightarrow \mathbb{R}$ with $g(l) \leq l$ for all $l \in \mathbb{N}$ and with $g(l) \rightarrow \infty$ as $l \rightarrow \infty$, each of the $n$-dimensional spaces $Z_{k}$ $(k \geq 1)$ will be constructed inside (any) 1-unconditional decomposition as above $F_{1} \oplus \cdots \oplus F_{N_{n}} \subset Y$, for some $N_{n} \in \mathbb{N}$, such that the following hold:

(a) $N_{n} \leq 3^{n}$

(b) Each $Z_{k}(k \geq 1)$ is spanned by seminormalized vectors $b_{1}^{(k)}, \ldots, b_{n}^{(k)}$ with

$$
1 \leq\left\|b_{i}^{(k)}\right\|<N_{n} \quad \text { for every } i=1, \ldots, n .
$$

Also, for all scalars $\alpha_{1}, \ldots, \alpha_{n}$ we have

$$
\text { (5) } \max \left(\left|\alpha_{1}\right|, \ldots,\left|\alpha_{n}\right|\right) \leq\left\|\alpha_{1} b_{1}^{(k)}+\cdots+\alpha_{n} b_{n}^{(k)}\right\|<N_{n}\left(\left|\alpha_{1}\right|+\cdots+\left|\alpha_{n}\right|\right) .
$$

In addition, the vectors $b_{1}^{(k)}, \ldots, b_{n}^{(k)} \in F_{1} \oplus \cdots \oplus F_{N_{n}}$ are supported on the corresponding $f_{1, k}, \ldots, f_{N_{n}, k}$ for all $k \geq 1$, with coefficients in $[0,1]$. They are defined based on the position of the indices $k$ with respect to the partition $\Delta_{N_{n}}=\left\{A_{N_{n}, l}\right\}_{l \geq 1}$, in the sense that their distributions depend on $g(l)$ whenever $k \in A_{N_{n}, l}$.

(c) Let $T: Y_{n} \rightarrow Y_{n}$ be a bounded linear operator such that $T\left(Z_{k}\right) \subset Z_{k}$, $T_{\mid Z_{k}}=\left[a_{i j}^{(k)}\right]_{1 \leq i, j \leq n}$ with respect to the basis of $Z_{k}$ for all $k \geq 1$. Then for every $l \geq 1$ there exists $k \in A_{N_{n}, l}$ such that

$$
\begin{aligned}
\max \left(\{ | a _ { i j } ^ { ( k ) } | : i \neq j \} \cup \left\{\left|a_{11}^{(k)}-a_{i i}^{(k)}\right|: 2\right.\right. & \leq i \leq n\}) \\
& \leq 3 C\left(N_{n}\right)\|T\| \cdot 2^{-g(l) /\left(3 \cdot 4^{N_{n}}\right)},
\end{aligned}
$$

where $C(N):=1+4+4^{2}+\cdots+4^{N-2}$.

(d) Let $N \geq 1$ and suppose that $\left(F_{1} \oplus \cdots \oplus F_{N_{n}}\right) \oplus\left(F_{N_{n}+1} \oplus \cdots \oplus F_{N_{n}+N}\right)$ $\subset Y$ is an unconditional decomposition of the type considered in this section. For every $k \geq 1$, let $b_{1}^{(k)}, \ldots, b_{n}^{(k)} \in F_{1} \oplus \cdots \oplus F_{N_{n}}$ be the same vectors as above. For every $l \geq 1$ and $k \in A_{N_{n}, l}$ consider the perturbations

$$
\widetilde{b}_{1}^{(k)}=b_{1}^{(k)}+2^{-g(l)} p_{1}^{(k)}, \ldots, \widetilde{b}_{n}^{(k)}=b_{n}^{(k)}+2^{-g(l)} p_{n}^{(k)},
$$

where $p_{1}^{(k)}, \ldots, p_{n}^{(k)} \in F_{N_{n}+1} \oplus \cdots \oplus F_{N_{n}+N}$ are vectors supported on the corresponding $f_{N_{n}+1, k}, \ldots, f_{N_{n}+N, k}$, with coefficients in $[0,1]$.

Let $T: \overline{\operatorname{span}}\left\{\widetilde{b}_{1}^{(k)}, \ldots, \widetilde{b}_{n}^{(k)}\right\}_{k \geq 1} \rightarrow F_{1} \oplus \cdots \oplus F_{N_{n}}$ be a bounded linear operator such that

$$
T \widetilde{b}_{j}^{(k)}=\sum_{i=1}^{n} a_{i j}^{(k)} b_{i}^{(k)}
$$

for every $j=1, \ldots, n$ and $k \geq 1$. Then for every $l \geq 1$ there exists $k \in A_{N_{n}, l}$ such that 


$$
\begin{aligned}
& \max \left(\left\{\left|a_{i j}^{(k)}\right|: i \neq j\right\} \cup\left\{\left|a_{11}^{(k)}-a_{i i}^{(k)}\right|: 2 \leq i \leq n\right\}\right) \\
& \leq 3 C\left(N_{n}+N\right)\|T\| \cdot 2^{-g(l) /\left(3 \cdot 4^{N_{n}}\right)} .
\end{aligned}
$$

(e) Similarly, let $\left(F_{1} \oplus \cdots \oplus F_{N}\right) \oplus\left(F_{N+1} \oplus \cdots \oplus F_{N+N_{n}}\right) \subset Y$ be a 1unconditional decomposition and, for every $k \geq 1$, let $b_{1}^{(k)}, \ldots, b_{n}^{(k)} \in F_{N+1} \oplus$ $\cdots \oplus F_{N+N_{n}}$ be the vectors discussed in (b) and (c), except that this time they are supported on $f_{N+1, k}, \ldots, f_{N+N_{n}, k}$. For every $k \geq 1$ let

$$
\widetilde{b}_{1}^{(k)}=p_{1}^{(k)}+b_{1}^{(k)}, \ldots, \widetilde{b}_{n}^{(k)}=p_{n}^{(k)}+b_{n}^{(k)},
$$

where now $p_{1}^{(k)}, \ldots, p_{n}^{(k)} \in F_{1} \oplus \cdots \oplus F_{N}$ are vectors supported on the corresponding $f_{1, k}, \ldots, f_{N, k}$, with coefficients in $[0,1]$.

As before, let $T: \overline{\operatorname{span}}\left\{\widetilde{b}_{1}^{(k)}, \ldots, \widetilde{b}_{n}^{(k)}\right\}_{k \geq 1} \rightarrow F_{N+1} \oplus \cdots \oplus F_{N+N_{n}}$ be a bounded linear operator satisfying (6). Then for every $l \geq 1$ there exist $k \in A_{N+N_{n}, l}$ such that (7) holds. Moreover, a similar statement is true when we consider the vectors $\widetilde{b}_{1}^{(k)}, \ldots, \widetilde{b}_{n}^{(k)}(k \geq 1)$ to be at the same time perturbations to the left and right of $b_{1}^{(k)}, \ldots, b_{n}^{(k)}$ (thus their definition is a combination of the ones above).

For every $n \geq 2$ the space $Y_{n}$ which satisfies (a)-(c) above will have a 1-unconditional decomposition into $n$-dimensional subspaces $\left\{Z_{k}\right\}_{k \geq 1}$; this follows from Remark 2.3, since each $Z_{k} \subset F_{1} \oplus \cdots \oplus F_{N_{n}}$ is supported on the corresponding $f_{1, k}, \ldots, f_{N_{n}, k}$ for all $k \geq 1$. On the other hand, we get $\mathcal{U}_{n-1}\left(Y_{n}\right)=\infty$. Otherwise take $T: Y_{n} \rightarrow Y_{n}$ as in Proposition 2.2 and on each $Z_{k}$, with $k \geq 1$, write $T_{\mid Z_{k}}$ in the matrix form with respect to the basis $\left\{b_{1}^{(k)}, \ldots, b_{n}^{(k)}\right\}$ as $T_{\mid Z_{k}}=\left[a_{i j}^{(k)}\right]_{1 \leq i, j \leq n}$. It is easy to see that (5) and Proposition 2.2(ii) imply

$$
\begin{aligned}
\max \left(\left\{\left|a_{i j}^{(k)}\right|: i \neq j\right\} \cup\left\{\left|a_{11}^{(k)}-a_{i i}^{(k)}\right|: 2 \leq i \leq n\right\}\right) & \\
& \geq \frac{1}{N_{n} n^{2}}\left\|T_{\mid Z_{k}}-a_{11}^{(k)} I_{Z_{k}}\right\| \geq \frac{1}{2 N_{n} n^{4}}
\end{aligned}
$$

for all $k \geq 1$. Combining this with (c) we get, for every $l \geq 1$,

$$
\|T\| \geq \frac{1}{6 N_{n} n^{4} C\left(N_{n}\right)} \cdot 2^{g(l) /\left(3 \cdot 4^{N}\right)},
$$

which is a contradiction since $g(l) \rightarrow \infty$ as $l \rightarrow \infty$.

For the actual recursive construction, at the initial step $n=2$ we start with $N_{2}=4$. Let $g: \mathbb{N} \rightarrow \mathbb{R}$ be an arbitrary function such that $g(l) \leq l$ for all $l \in \mathbb{N}$, and let $F_{1} \oplus \cdots \oplus F_{4} \subset Y$ be a decomposition satisfying (2) and (3). For $k=1,2, \ldots$ the vectors $b_{1}^{(k)}, b_{2}^{(k)} \in F_{1} \oplus \cdots \oplus F_{4}$ will be defined 
similarly to [9] (see also [14]). That is, for $k \in A_{4, l}$ and $l=1,2, \ldots$ put

$$
\begin{aligned}
b_{1}^{(k)}=f_{1, k}+\quad+2^{-g(l) / 3} f_{3, k} & +2^{-2 g(l) / 3} f_{4, k}, \\
b_{2}^{(k)}= & f_{2, k}+\quad+2^{-2 g(l) / 3} f_{4, k} .
\end{aligned}
$$

It is clear that such vectors satisfy the requirement (b). They were designed to satisfy (c) and this is one of the main points of the arguments from [9], [14]. As a consequence of the same line of argument we will now show that the more general statements (d) and (e) are true as well.

We start with $(\mathrm{d})$, so let $\left(F_{1} \oplus \cdots \oplus F_{4}\right) \oplus\left(F_{5} \oplus \cdots \oplus F_{N+4}\right)$ be a decomposition of the type considered in this section. For each $l \geq 1$ and $k \in A_{4, l}$, define $b_{1}^{(k)}$ and $b_{2}^{(k)}$ as in (8) and then consider

$$
\widetilde{b}_{1}^{(k)}=b_{1}^{(k)}+2^{-g(l)} p_{1}^{(k)}, \quad \widetilde{b}_{2}^{(k)}=b_{2}^{(k)}+2^{-g(l)} p_{2}^{(k)},
$$

where $p_{1}^{(k)}$ and $p_{2}^{(k)}$ are vectors supported on the corresponding $f_{5, k}, \ldots$, $f_{N+4, k}$, whose coefficients belong to $[0,1]$.

Let $T: \overline{\operatorname{span}}\left\{\tilde{b}_{1}^{(k)}, \widetilde{b}_{2}^{(k)}\right\}_{k \geq 1} \rightarrow F_{1} \oplus \cdots \oplus F_{4}$ be a bounded linear operator such that for every $k \geq 1$,

$$
T \widetilde{b}_{1}^{(k)}=a_{k} b_{1}^{(k)}+c_{k} b_{2}^{(k)}, \quad T \widetilde{b}_{2}^{(k)}=b_{k} b_{1}^{(k)}+d_{k} b_{2}^{(k)} .
$$

In order to verify (7) fix an arbitrary $l \geq 1$. If we let $\mathcal{M}_{i}=\left\{m: A_{i, m} \subset A_{4, l}\right\}$ for $i=1,2,3$, then $\min \mathcal{M}_{i} \geq l \geq g(l)$ since $\Delta_{1} \succ \cdots \succ \Delta_{4}$.

We start by picking a set $B \subset A_{4, l}$ such that $\left|B \cap A_{1, m}\right|=1$ for all $m \in \mathcal{M}_{1}$; in other words, $B \cap A_{2, m} \in \mathcal{K}_{2, m}$ for all $m \in \mathcal{M}_{2}$.

According to (3), for every $m \in \mathcal{M}_{2}$, there exist scalars $\left\{\alpha_{k}\right\}_{k \in B \cap A_{2, m}}$ such that $\left\|\sum_{k \in B \cap A_{2, m}} \alpha_{k} f_{1, k}\right\|=1$ and $\left\|\sum_{k \in B \cap A_{2, m}} \alpha_{k} f_{2, k}\right\| \geq 2^{m}$. Since $\left\{f_{1, k}\right\}_{k}$ and $\left\{f_{2, k}\right\}_{k}$ are normalized, continuity yields scalars $\left\{\beta_{k}\right\}_{k \in B \cap A_{2, m}}$ such that $\left\|\sum_{k \in B \cap A_{2, m}} \beta_{k} f_{1, k}\right\|=1$ and $\left\|\sum_{k \in B \cap A_{2, m}} \beta_{k} f_{2, k}\right\|=2^{g(l) / 3}$.

Using the particular form of the vectors $\left\{p_{1}^{(k)}\right\}_{k}$, property (2) of the partitions $\Delta_{1}, \ldots, \Delta_{N+4}$ and unconditionality we get

$$
\begin{aligned}
\left\|\sum_{k \in B \cap A_{2, m}} \beta_{k} \widetilde{b}_{1}^{(k)}\right\| & \left\|\sum_{k \in B \cap A_{2, m}} \beta_{k} f_{1, k}\right\|+2^{-g(l) / 3}\left\|\sum_{k \in B \cap A_{2, m}} \beta_{k} f_{3, k}\right\| \\
& +2^{-2 g(l) / 3}\left\|\sum_{k \in B \cap A_{2, m}} \beta_{k} f_{4, k}\right\|+2^{-g(l)}\left\|\sum_{k \in B \cap A_{2, m}} \beta_{k} p_{1}^{(k)}\right\|
\end{aligned}
$$




$$
\begin{aligned}
\leq & 1+\left(4 \cdot 2^{-g(l) / 3}+4^{2} \cdot 2^{-2 g(l) / 3}\right)\left\|\sum_{k \in B \cap A_{2, m}} \beta_{k} f_{2, k}\right\| \\
& +2^{-g(l)} \sum_{i=5}^{N+4}\left\|\sum_{k \in B \cap A_{2, m}} \beta_{k} f_{i, k}\right\| \\
\leq & 1+\left[4 \cdot 2^{-g(l) / 3}+4^{2} \cdot 2^{-2 g(l) / 3}\right. \\
& \left.+2^{-g(l)}\left(4^{3}+\cdots+4^{N+2}\right)\right]\left\|\sum_{k \in B \cap A_{2, m}} \beta_{k} f_{2, k}\right\| \\
\leq & 1+4+4^{2}+\cdots+4^{N+2}=C(N+4) .
\end{aligned}
$$

On the other hand,

$$
\begin{aligned}
\left\|T\left(\sum_{k \in B \cap A_{2, m}} \beta_{k} \widetilde{b}_{1}^{(k)}\right)\right\| & =\left\|\sum_{k \in B \cap A_{2, m}} \beta_{k}\left(a_{k} b_{1}^{(k)}+c_{k} b_{2}^{(k)}\right)\right\| \\
& \geq\left\|\sum_{k \in B \cap A_{2, m}} \beta_{k} c_{k} f_{2, k}\right\| \\
& \geq \min _{k \in B \cap A_{2, m}}\left|c_{k}\right|\left\|\sum_{k \in B \cap A_{2, m}} \beta_{k} f_{2, k}\right\| \\
& \geq 2^{g(l) / 3} \min _{k \in B \cap A_{2, m}}\left|c_{k}\right|
\end{aligned}
$$

and thus for every $m \in \mathcal{M}_{2}$ there exists $k \in B \cap A_{2, m}$ such that

$$
\left|c_{k}\right| \leq C(N+4)\|T\| \cdot 2^{-g(l) / 3} .
$$

Let $B^{\prime} \subset B$ be the collection of such indices $k$ as above. Then, for every $m \in \mathcal{M}_{3}$, we get $B^{\prime} \cap A_{3, m} \in \mathcal{K}_{3, m}$ and thus by (3) there exist scalars $\left\{\beta_{k}\right\}_{k \in B^{\prime} \cap A_{3, m}}$ such that

$$
\left\|\sum_{k \in B^{\prime} \cap A_{3, m}} \beta_{k}\left(f_{1, k}+f_{2, k}\right)\right\|=1, \quad\left\|\sum_{k \in B^{\prime} \cap A_{3, m}} \beta_{k} f_{3, k}\right\|=2^{2 g(l) / 3} .
$$

In much the same way as before,

$$
\left\|\sum_{k \in B^{\prime} \cap A_{3, m}} \beta_{k} \widetilde{b}_{2}^{(k)}\right\|
$$

$$
\begin{aligned}
& \leq\left\|\sum_{k \in B^{\prime} \cap A_{3, m}} \beta_{k} f_{2, k}\right\|+2^{-2 g(l) / 3}\left\|_{k \in B^{\prime} \cap A_{3, m}} \beta_{k} f_{4, k}\right\|+2^{-g(l)}\left\|\sum_{k \in B^{\prime} \cap A_{3, m}} \beta_{k} p_{2}^{(k)}\right\| \\
& \leq 1+\left[4 \cdot 2^{-2 g(l) / 3}+2^{-g(l)}\left(4^{2}+\cdots+4^{N+1}\right)\right]\left\|\sum_{k \in B^{\prime} \cap A_{3, m}} \beta_{k} f_{3, k}\right\| \leq C(N+4)
\end{aligned}
$$


while

$$
\begin{aligned}
\left\|T\left(\sum_{k \in B^{\prime} \cap A_{3, m}} \beta_{k} \widetilde{b}_{2}^{(k)}\right)\right\| & =\left\|\sum_{k \in B^{\prime} \cap A_{3, m}} \beta_{k}\left(b_{k} b_{1}^{(k)}+d_{k} b_{2}^{(k)}\right)\right\| \\
& \geq 2^{-g(l) / 3}\left\|\sum_{k \in B^{\prime} \cap A_{3, m}} \beta_{k} b_{k} f_{3, k}\right\| \\
& \geq 2^{g(l) / 3} \min _{k \in B^{\prime} \cap A_{3, m}}\left|b_{k}\right| .
\end{aligned}
$$

For every $m \in \mathcal{M}_{3}$ we can take some $k \in B^{\prime} \cap A_{3, m}$ such that

$$
\left|b_{k}\right| \leq C(N+4)\|T\| \cdot 2^{-g(l) / 3}
$$

and then define $B^{\prime \prime} \subset B^{\prime} \subset B$ to be the set of such indices $k$.

Again we can argue that $B^{\prime \prime} \in \mathcal{K}_{4, l}$ and therefore we can pick scalars $\left\{\beta_{k}\right\}_{k \in B^{\prime \prime}}$ satisfying

$$
\left\|\sum_{k \in B^{\prime \prime}} \beta_{k}\left(f_{1, k}+f_{2, k}+f_{3, k}\right)\right\|=1, \quad\left\|\sum_{k \in B^{\prime \prime}} \beta_{k} f_{4, k}\right\|=2^{g(l)} .
$$

It is not hard to see that

$$
\left\|\sum_{k \in B^{\prime \prime}} \beta_{k}\left(\widetilde{b}_{1}^{(k)}-\widetilde{b}_{2}^{(k)}\right)\right\| \leq C(N+4)
$$

and

$$
\begin{aligned}
\left\|T\left(\sum_{k \in B^{\prime \prime}} \beta_{k}\left(\widetilde{b}_{1}^{(k)}-\widetilde{b}_{2}^{(k)}\right)\right)\right\| & \geq 2^{-2 g(l) / 3 \|}\left\|\sum_{k \in B^{\prime \prime}} \beta_{k}\left(\left(a_{k}-b_{k}\right)+\left(c_{k}-d_{k}\right)\right) f_{4, k}\right\| \\
& \geq 2^{g(l) / 3} \min _{k \in B^{\prime \prime}}\left|\left(a_{k}-b_{k}\right)+\left(c_{k}-d_{k}\right)\right| .
\end{aligned}
$$

For $k \in B^{\prime \prime}$ such that $\left|\left(a_{k}-b_{k}\right)+\left(c_{k}-d_{k}\right)\right| \leq C(N+4)\|T\| \cdot 2^{-g(l) / 3}$ we find, taking into account (11) and (13), that $\left|a_{k}-d_{k}\right| \leq 3 C(N+4)\|T\| \cdot 2^{-g(l) / 3}$, which shows that $(d)$ is satisfied for the initial step of induction (with a slightly better estimate than $(7))$.

In order to check $(\mathrm{e})$, let $\left(F_{1} \oplus \cdots \oplus F_{N}\right) \oplus\left(F_{N+1} \oplus \cdots \oplus F_{N+4}\right)$ be an unconditional decomposition which satisfies (2) and (3) for $i=1, \ldots, N+3$. Let $g: \mathbb{N} \rightarrow \mathbb{R}$ be a function such that $g(l) \leq l$ for all $l \in \mathbb{N}$.

For $l \geq 1$ and $k \in A_{N+4, l}$ define the vectors $b_{1}^{(k)}, b_{2}^{(k)} \in F_{N+1} \oplus \cdots \oplus F_{N+4}$ as in (8), except that this time they are supported on $f_{N+1, k}, \ldots, f_{N+4, k}$, and let

$$
\widetilde{b}_{1}^{(k)}=p_{1}^{(k)}+b_{1}^{(k)}, \quad \widetilde{b}_{2}^{(k)}=p_{2}^{(k)}+b_{2}^{(k)},
$$

where $p_{1}^{(k)}$ and $p_{1}^{(k)}$ are vectors supported on the corresponding $f_{1, k}, \ldots, f_{N, k}$ with coefficients in $[0,1]$.

Let $T: \overline{\operatorname{span}}\left\{\widetilde{b}_{1}^{(k)}, \widetilde{b}_{2}^{(k)}\right\}_{k \geq 1} \rightarrow F_{N+1} \oplus \cdots \oplus F_{N+4}$ be a bounded operator satisfying (9) for every $k \geq 1$. The fact that for each $l \geq 1$ there exists 
$k \in A_{N+4, l}$ such that (7) is satisfied follows in the same way as in (d). The analysis is now done inside $F_{N+1} \oplus \cdots \oplus F_{N+4}$. To see how the estimates corresponding to (10), (12) and (14) are obtained, pick a set $B \subset A_{N+4, l}$ which intersects all the appropriate members of the partition $\Delta_{N+1}$ and for every suitable $m \geq 1$ take scalars $\left\{\beta_{k}\right\}_{k \in B \cap A_{N+2, m}}$ such that

$$
\left\|\sum_{k \in B \cap A_{N+2, m}} \beta_{k}\left(f_{1, k}+\cdots+f_{N+1, k}\right)\right\|=1, \quad\left\|\sum_{k \in B \cap A_{N+2, m}} \beta_{k} f_{N+2, k}\right\|=2^{g(l) / 3} .
$$

Then the unconditionality implies

$$
\begin{aligned}
& \left\|\sum_{k \in B \cap A_{N+2, m}} \beta_{k} \widetilde{b}_{1}^{(k)}\right\| \\
& \leq\left\|\sum_{k \in B \cap A_{N+2, m}} \beta_{k} p_{1}^{(k)}\right\|+\left\|\sum_{k \in B \cap A_{N+2, m}} \beta_{k} f_{N+1, k}\right\| \\
& \quad+2^{-g(t) / 3}\left\|_{k \in B \cap A_{N+2, m}} \sum_{k} f_{N+3, k}\right\|+2^{-2 g(t) / 3}\left\|_{k \in B \cap A_{N+2, m}} \beta_{k} f_{N+4, k}\right\| \\
& \leq \sum_{i=1}^{N}\left\|\sum_{k \in B \cap A_{N+2, m}} \beta_{k} f_{i, k}\right\|+1 \\
& \quad+\left(4 \cdot 2^{-g(t) / 3}+4^{2} \cdot 2^{-2 g(t) / 3}\right)\left\|\sum_{k \in B \cap A_{N+2, m}} \beta_{k} f_{N+2, k}\right\| \\
& \leq N+21 \leq C(N+4),
\end{aligned}
$$

thus the estimate corresponding to (10). We can deal with (12) and (14) in a similar way and then (e) will follow (again with a slightly better estimate).

For the last statement of (e) when we have to consider, for $M, L \in \mathbb{N}$, vectors $\widetilde{b}_{1}^{(k)}, \widetilde{b}_{2}^{(k)} \in\left(F_{1} \oplus \cdots \oplus F_{M}\right) \oplus\left(F_{M+1} \oplus \cdots \oplus F_{M+4}\right) \oplus\left(F_{M+5} \oplus \cdots \oplus\right.$ $\left.F_{M+L+4}\right)$ which are at the same time perturbations to the left and right of $b_{1}^{(k)}, b_{2}^{(k)} \in F_{M+1} \oplus \cdots \oplus F_{M+4}(k \geq 1)$, the estimates are obtained by combining the ones above; as a result we get $k \in A_{M+4, l}$ with

$$
\begin{aligned}
\max \left\{\left|a_{k}-d_{k}\right|,\left|b_{k}\right|,\left|c_{k}\right|\right\} & \leq 3(C(M+4)+C(L+4))\|T\| \cdot 2^{-g(l) / 3} \\
& \leq 3 C(M+L+4)\|T\| \cdot 2^{-g(l) / 3} .
\end{aligned}
$$

Inductively, assume that we have constructed the required vectors for some $n \geq 2$. Let $g: \mathbb{N} \rightarrow \mathbb{R}$ be a function such that $g(l) \leq l$ for $l \in \mathbb{N}$. For the next step of the construction we will work inside (any) unconditional decomposition $\left(F_{1} \oplus \cdots \oplus F_{N_{n}}\right) \oplus\left(F_{N_{n}+1} \oplus \cdots \oplus F_{N_{n}+4}\right) \oplus\left(F_{N_{n}+5} \oplus \cdots \oplus\right.$ $\left.F_{2 N_{n}+4}\right) \subset Y$ that satisfies $(2)$ and (3). Thus $N_{n+1}=2 N_{n}+4$. 
For $k=1,2, \ldots$ let $u_{1}^{(k)}, \ldots, u_{n}^{(k)} \in F_{N_{n}+5} \oplus \cdots \oplus F_{N_{n+1}}$ be vectors obtained at the previous inductive step corresponding to the function $g$ (we changed the notation for such vectors to avoid the possible confusion with the new vectors that are to be constructed). Thus they are defined based on the position of $k$ with respect to the partition $\left\{A_{N_{n+1}, l}\right\}_{l \geq 1}$ and their distributions depend on the corresponding value of $g(l)$.

For an arbitrary $l \geq 1$ let

$$
\mathcal{M}_{1, l}=\left\{s: A_{N_{n}+4, s} \subset A_{N_{n+1}, l}\right\}, \quad \mathcal{M}_{2, l}=\left\{s: A_{N_{n}, s} \subset A_{N_{n+1}, l}\right\} .
$$

For $s=1,2, \ldots$ and $k \in A_{N_{n+4}, s}$, let $x_{k}, y_{k} \in F_{N_{n}+1} \oplus \cdots \oplus F_{N_{n}+4}$ be vectors obtained at the inductive step $n=2$ (similarly to (8)), defined using the function $\widetilde{g}: \mathbb{N} \rightarrow \mathbb{R}, \widetilde{g}(s):=g(l) / 4^{N_{n}+1}$ for every $s \in \mathcal{M}_{1, l}$ and every $l \geq 1$. Notice that if $l$ is such that $s \in \mathcal{M}_{1, l}$ then

$$
\widetilde{g}(s) \leq g(l) \leq l \leq s .
$$

Finally, for $s=1,2, \ldots$ and $k \in A_{N_{n}, s}$, let $v_{1}^{(k)}, \ldots, v_{n}^{(k)} \in F_{1} \oplus \cdots \oplus F_{N_{n}}$ be vectors obtained at the previous inductive step, corresponding to the function $\widetilde{\widetilde{g}}: \mathbb{N} \rightarrow \mathbb{R}, \widetilde{\widetilde{g}}(s):=g(l) / 4^{N_{n}+2}$ whenever $s \in \mathcal{M}_{2, l}$ (with $l \geq 1$ ). If $l$ is such that $s \in \mathcal{M}_{2, l}$ then again

$$
\widetilde{\widetilde{g}}(s) \leq g(l) \leq l \leq s .
$$

Now, for $l=1,2, \ldots$ and $k \in A_{N_{n+1}, l}$ define the following vectors inside $\left(F_{1} \oplus \cdots \oplus F_{N_{n}}\right) \oplus\left(F_{N_{n}+1} \oplus \cdots \oplus F_{N_{n}+4}\right) \oplus\left(F_{N_{n}+5} \oplus \cdots \oplus F_{N_{n+1}}\right):$

$$
\begin{array}{rlrl}
b_{1}^{(k)} & =v_{1}^{(k)}+2^{-g(l) / 4^{N_{n}+2}} x_{k}, & \\
b_{2}^{(k)} & =v_{2}^{(k)}+ & & \\
& \vdots & & \\
b_{n}^{(k)} & =v_{n}^{(k)}+ & & \\
b_{n+1}^{(k)} & = & y_{k}+2^{-g(l) / 4^{N_{n}+1}} u_{1}^{(k)}, & 2^{-g(l) / 4^{N_{n}+1}+1} u_{n-1}^{(k)} u_{n}^{(k)} .
\end{array}
$$

Let us now check that conditions (b)-(e) are satisfied.

By definition, it is clear that for every $k=1,2, \ldots$ the vectors $b_{1}^{(k)}, \ldots$, $b_{n+1}^{(k)}$ are supported on $f_{1, k}, \ldots, f_{N_{n+1}, k}$. If $\alpha_{1}, \ldots, \alpha_{n+1}$ are scalars then by the unconditionality and previous inductive steps

$$
\begin{aligned}
& \left\|\alpha_{1} b_{1}^{(k)}+\cdots+\alpha_{n+1} b_{n+1}^{(k)}\right\| \\
& \quad \geq \max \left\{\left\|\alpha_{1} v_{1}^{(k)}+\cdots+\alpha_{n} v_{n}^{(k)}\right\|,\left\|\alpha_{1} \cdot 2^{-g(l) / 4^{N_{n}+2}} x_{k}+\alpha_{n+1} y_{k}\right\|\right\} \\
& \quad \geq \max \left\{\left|\alpha_{1}\right|, \ldots,\left|\alpha_{n}\right|,\left|\alpha_{n+1}\right|\right\} .
\end{aligned}
$$

Regarding (c) and (d) (and also (e)), at every step of the induction we will actually prove the following slightly more general statements: for every $l \geq 1$ and every set $B_{l} \subset A_{N_{n}, l}$ (respectively $B_{l} \subset A_{N_{n}+N, l}$ ) such 
that $B_{l} \cap A_{1, m} \neq \emptyset$ whenever $A_{1, m} \subset A_{N_{n}, l}$ (respectively $B_{l} \cap A_{N+1, m} \neq \emptyset$ whenever $A_{N+1, m} \subset A_{N_{n}+N, l}$ ) there exists $k \in B_{l}$ such that the estimates of (c) and (d) (respectively (e)) are satisfied. For the first step of the induction these statements are valid as can be seen from the previous arguments.

We start with (c), so let $Z_{k}=\operatorname{span}\left\{b_{1}^{(k)}, \ldots, b_{n+1}^{(k)}\right\}$ for $k \geq 1$, and let $T: \overline{\operatorname{span}}\left\{Z_{k}\right\}_{k \geq 1} \rightarrow \overline{\operatorname{span}}\left\{Z_{k}\right\}_{k \geq 1}$ be a bounded linear operator such that $T\left(Z_{k}\right) \subset Z_{k}, T_{\mid Z_{k}}=\left[a_{i j}^{(k)}\right]_{1 \leq i, j \leq n+1}$ with respect to the basis of $Z_{k}$ for $k=1,2, \ldots$.

Fix an arbitrary $l \geq 1$ and take $B_{l} \subset A_{N_{n+1}, l}$ such that $B_{l} \cap A_{1, m} \neq \emptyset$ whenever $A_{1, m} \subset A_{N_{n+1}, l}$.

It is easy to see that we can apply the inductive hypothesis (d) for the vectors $v_{1}^{(k)}, \ldots, v_{n}^{(k)} \in F_{1} \oplus \cdots \oplus F_{N_{n}}$ (with $k \geq 1$ ) and the operator $P_{F_{1} \oplus \cdots \oplus F_{N_{n}}} T_{\mid \overline{\operatorname{span}}\left\{b_{1}^{(k)}, \ldots, b_{n}^{(k)}\right\}_{k \geq 1}}: \overline{\operatorname{span}}\left\{b_{1}^{(k)}, \ldots, b_{n}^{(k)}\right\}_{k \geq 1} \rightarrow F_{1} \oplus \cdots \oplus F_{N_{n}}$. Thus for every $s \in \mathcal{M}_{2, l}$ there is $k_{s} \in B_{l} \cap A_{N_{n}, s}$ such that

$$
\begin{aligned}
& \max \left(\left\{\left|a_{i j}^{\left(k_{s}\right)}\right|: i \neq j, i, j \in\{1, \ldots, n\}\right\} \cup\left\{\left|a_{11}^{\left(k_{s}\right)}-a_{i i}^{\left(k_{s}\right)}\right|: 2 \leq i \leq n\right\}\right) \\
& \quad \leq 3 C\left(N_{n+1}\right)\|T\| \cdot 2^{-\widetilde{\widetilde{g}}(s) /\left(3 \cdot 4^{N_{n}}\right)}=3 C\left(N_{n+1}\right)\|T\| \cdot 2^{-g(l) /\left(3 \cdot 4^{2 N_{n}+2}\right)} \\
& \quad \leq 3 C\left(N_{n+1}\right)\|T\| \cdot 2^{-g(l) /\left(3 \cdot 4^{N_{n+1}}\right)} .
\end{aligned}
$$

Let $B_{l}^{\prime} \subset B_{l}$ be the collection of all such indices $k$ as above. It is clear that $B_{l}^{\prime} \cap A_{N_{n}+1, m} \neq \emptyset$ whenever $A_{N_{n}+1, m} \subset A_{N_{n+1}, l}$. We will now invoke (e) for the initial inductive step, applied to the perturbations (both to the left and right) of the vectors $x_{k}, y_{k} \in F_{N_{n}+1} \oplus \cdots \oplus F_{N_{n}+4}$ (with $k=1,2, \ldots$ ) and the operator $P_{F_{N_{n}+1} \oplus \cdots \oplus F_{N_{n}+4}} T_{\mid \overline{\operatorname{span}}\left\{b_{1}^{(k)}, b_{n+1}^{(k)}\right\}_{k \geq 1}}: \overline{\operatorname{span}}\left\{b_{1}^{(k)}, b_{n+1}^{(k)}\right\}_{k \geq 1} \rightarrow F_{N_{n}+1}$ $\oplus \cdots \oplus F_{N_{n}+4}$. Then we deduce that for every $s \in \mathcal{M}_{1, l}$ there exists $k_{s} \in$ $B_{l}^{\prime} \cap A_{N_{n}+4, s}$ such that, taking into account (11), (13),

$$
\begin{aligned}
\max \left\{\left|a_{1, n+1}^{\left(k_{s}\right)}\right|, \mid a_{n+1,1}^{\left(k_{s}\right)}\right. & \left.|,| a_{11}^{\left(k_{s}\right)}-a_{n+1, n+1}^{\left(k_{s}\right)} \mid\right\} \\
& \leq 3 C\left(N_{n+1}\right)\|T\| \cdot 2^{-\widetilde{g}(s) / 3} \cdot 2^{g(l) / 4^{N_{n}+2}} \\
& \leq 3 C\left(N_{n+1}\right)\|T\| \cdot 2^{-g(l) /\left(3 \cdot 4^{N_{n}+1}\right)} \cdot 2^{g(l) / 4^{N_{n}+2}} \\
& \leq 3 C\left(N_{n+1}\right)\|T\| \cdot 2^{-g(l) /\left(3 \cdot 4^{N_{n}+2}\right)} .
\end{aligned}
$$

The fact that an extra constant $2^{g(l) / 4^{N_{n}+2}}$ appears in these estimates is due to the specific form of the vectors involved and it follows easily from the arguments corresponding to the initial inductive step $n=2$; more specifically, this extra constant will have no influence on the estimates from above that appear there, it will only be a part of the estimates from below.

Finally, let $B_{l}^{\prime \prime} \subset B_{l}^{\prime}\left(\subset B_{l}\right)$ be the set of all indices $k$ for which (16) is satisfied. Applying the inductive hypothesis (e) to the vectors $u_{1}^{(k)}, \ldots, u_{n}^{(k)} \in$ 
$F_{N_{n}+5} \oplus \cdots \oplus F_{N_{n+1}}($ with $k \geq 1)$ and $P_{F_{N_{n}+5} \oplus \cdots \oplus F_{N_{n+1}}} T_{\mid \overline{\operatorname{span}}\left\{b_{2}^{(k)}, \ldots, b_{n+1}^{(k)}\right\}_{k \geq 1}}$ : $\overline{\operatorname{span}}\left\{b_{2}^{(k)}, \ldots, b_{n+1}^{(k)}\right\}_{k \geq 1} \rightarrow F_{N_{n}+5} \oplus \cdots \oplus F_{N_{n+1}}$ we get $k \in B_{l}^{\prime \prime}$ such that $\max \left\{\left|a_{i j}^{(k)}\right|: i \neq j, i, j \in\{2, \ldots, n+1\}\right\}$ $\leq 3 C\left(N_{n+1}\right)\|T\| \cdot 2^{-g(l) /\left(3 \cdot 4^{N_{n}}\right)} \cdot 2^{g(l) / 4^{N_{n}+1}} \leq 3 C\left(N_{n+1}\right)\|T\| \cdot 2^{-g(l) /\left(3 \cdot 4^{N_{n}+1}\right)}$. Combining this with (15) and (16) we get the conclusion of (c).

In order to prove (d) and (e) we can apply the same arguments as before for the perturbation vectors $\widetilde{b}_{1}^{(k)}, \ldots, \widetilde{b}_{n+1}^{(k)} \in F_{1} \oplus \cdots \oplus F_{N_{n+1}+N}$, instead of $b_{1}^{(k)}, \ldots, b_{n+1}^{(k)}$, for $k \geq 1$. The computations will be completely similar to (15), (16), the only difference being the replacement of the constant $C\left(N_{n+1}\right)$ with $C\left(N_{n+1}+N\right)$ due to the nature of the vectors considered.

4. Recursive construction in case (II). Let $Y$ be a Banach space with a 1-unconditional basis. Suppose that $Y$ does not contain a subspace isomorphic to $\ell_{2}$ and that it has finite cotype.

As in Section 3, for every $n \geq 2$ we will construct a subspace $Y_{n} \subset Y$ with the property that $\mathcal{U}_{n-1}(Y)=\infty$, this time inside (any) decomposition $F_{1} \oplus \cdots \oplus F_{N_{n}} \subset Y$, for some $N_{n} \in \mathbb{N}$, which satisfies the conditions (2) and (4). The space $Y_{n}$ will have a 1-unconditional decomposition into $n$ dimensional subspaces $\left\{Z_{k}\right\}_{k \geq 1}$, where for every $k \geq 1$ the space $Z_{k}$ is spanned by vectors $b_{1}^{(k)}, \ldots, b_{n}^{(k)}$ with the following properties:

(a) $N_{n} \leq 3^{n}$.

(b) The vectors $b_{1}^{(k)}, \ldots, b_{n}^{(k)} \in F_{1} \oplus \cdots \oplus F_{N_{n}}$ are supported on the corresponding $f_{1, k}, \ldots, f_{N_{n}, k}$ for all $k \geq 1$, with coefficients in [0,1]. In addition, for all scalars $\alpha_{1}, \ldots, \alpha_{n}$,

$$
\frac{1}{2} \max \left(\left|\alpha_{1}\right|, \ldots,\left|\alpha_{n}\right|\right) \leq\left\|\alpha_{1} b_{1}^{(k)}+\cdots+\alpha_{n} b_{n}^{(k)}\right\|<N_{n}\left(\left|\alpha_{1}\right|+\cdots+\left|\alpha_{n}\right|\right) .
$$

(c) Let $T: Y_{n} \rightarrow Y_{n}$ be a bounded linear operator such that $T\left(Z_{k}\right) \subset Z_{k}$, $T_{\mid Z_{k}}=\left[a_{i j}^{(k)}\right]_{1 \leq i, j \leq n}$ with respect to the basis of $Z_{k}$ for all $k \geq 1$. Then for every $l \geq 1$ there is $k \in A_{N_{n}, l}$ such that

$$
\max \left(\left\{\left|a_{i j}^{(k)}\right|: i \neq j\right\} \cup\left\{\left|a_{11}^{(k)}-a_{i i}^{(k)}\right|: 2 \leq i \leq n\right\}\right) \leq 3 C\left(N_{n}\right)\|T\| \cdot 2^{-l / 2}
$$

where, as before, $C(N):=1+4+4^{2}+\cdots+4^{N-2}$.

(d) Let $N \geq 1$ and suppose that $\left(F_{1} \oplus \cdots \oplus F_{N_{n}}\right) \oplus\left(F_{N_{n}+1} \oplus \cdots \oplus\right.$ $\left.F_{N_{n}+N}\right) \subset Y$ is a decomposition of the type considered in this section. Let $b_{1}^{(k)}, \ldots, b_{n}^{(k)} \in F_{1} \oplus \cdots \oplus F_{N_{n}}$ be the same vectors as above, for every $k \geq 1$, and consider the perturbations

$$
\widetilde{b}_{1}^{(k)}=b_{1}^{(k)}+p_{1}^{(k)}, \ldots, \widetilde{b}_{n}^{(k)}=b_{n}^{(k)}+p_{n}^{(k)},
$$


where $p_{1}^{(k)}, \ldots, p_{n}^{(k)} \in F_{N_{n}+1} \oplus \cdots \oplus F_{N_{n}+N}$ are vectors supported on the corresponding $f_{N_{n}+1, k}, \ldots, f_{N_{n}+N, k}$ with coefficients in $[0,1]$.

If $T: \overline{\operatorname{span}}\left\{\widetilde{b}_{1}^{(k)}, \ldots, \widetilde{b}_{n}^{(k)}\right\}_{k \geq 1} \rightarrow F_{1} \oplus \cdots \oplus F_{N_{n}}$ is a bounded linear operator satisfying (6) then for every $l \geq 1$ there is $k \in A_{N_{n}, l}$ such that

$$
\begin{aligned}
\max \left(\{ | a _ { i j } ^ { ( k ) } | : i \neq j \} \cup \left\{\left|a_{11}^{(k)}-a_{i i}^{(k)}\right|: 2\right.\right. & \leq i \leq n\}) \\
& \leq 3 C\left(N_{n}+N\right)\|T\| \cdot 2^{-l / 2} .
\end{aligned}
$$

(e) Similarly to (d), let $\left(F_{1} \oplus \cdots \oplus F_{N}\right) \oplus\left(F_{N+1} \oplus \cdots \oplus F_{N+N_{n}}\right) \subset Y$ and, for $k \geq 1$, let $b_{1}^{(k)}, \ldots, b_{n}^{(k)} \in F_{N+1} \oplus \cdots \oplus F_{N+N_{n}}$ be the vectors discussed in (b), except that this time they are supported on $f_{N+1, k}, \ldots, f_{N+N_{n}, k}$.

For every $r \geq 1$ and $k \in A_{N+1, r}$ let

$$
\widetilde{b}_{1}^{(k)}=2^{-r} p_{1}^{(k)}+b_{1}^{(k)}, \ldots, \widetilde{b}_{n}^{(k)}=2^{-r} p_{n}^{(k)}+b_{n}^{(k)},
$$

where now $p_{1}^{(k)}, \ldots, p_{n}^{(k)} \in F_{1} \oplus \cdots \oplus F_{N}$ are vectors supported on the corresponding $f_{1, k}, \ldots, f_{N, k}$ with coefficients in $[0,1]$.

Then for any bounded linear operator $T: \overline{\operatorname{span}}\left\{\widetilde{b}_{1}^{(k)}, \ldots, \widetilde{b}_{n}^{(k)}\right\}_{k \geq 1} \rightarrow$ $F_{N+1} \oplus \cdots \oplus F_{N+N_{n}}$ which satisfies (6) and for every $l \geq 1$ there exists $k \in A_{N+N_{n}, l}$ such that (18) holds. In addition, if we consider the vectors $\widetilde{b}_{1}^{(k)}, \ldots, \widetilde{b}_{n}^{(k)}(k \geq 1)$ to be at the same time perturbations to the left and right of $b_{1}^{(k)}, \ldots, b_{n}^{(k)}$ (and therefore a combination of (17) and (19)), we obtain a similar result.

For the first step of the induction, $n=2$, we put $N_{2}=4$ and we follow the construction from [9], [14]. For every $k \in A_{2, s} \cap A_{3, t}$, with $s=1,2, \ldots$. and $t=1,2, \ldots$, define

$$
\begin{array}{cc}
b_{1}^{(k)}= & 2^{-t / 2} f_{2, k}+f_{3, k}+f_{4, k}, \\
b_{2}^{(k)}=2^{-s / 2} f_{1, k} & +f_{3, k} .
\end{array}
$$

We will only discuss the proof of (e), since (c) and (d) follow in a similar manner (actually, (c) is a particular case of $(\mathrm{e}))$. Thus let $\left(F_{1} \oplus \cdots \oplus F_{N}\right)$ $\oplus\left(F_{N+1} \oplus \cdots \oplus F_{N+N_{n}}\right) \subset Y$ be a suitable decomposition, of the type considered in this section. Having defined, for all $k \geq 1$, the vectors $b_{1}^{(k)}, b_{2}^{(k)} \in$ $F_{N+1} \oplus \cdots \oplus F_{N+4}$ similarly to (20), we let, for every $r \geq 1$ and $k \in A_{N+1, r}$,

$$
\widetilde{b}_{1}^{(k)}=2^{-r} p_{1}^{(k)}+b_{1}^{(k)}, \quad \widetilde{b}_{2}^{(k)}=2^{-r} p_{2}^{(k)}+b_{2}^{(k)},
$$

with $p_{1}^{(k)}, p_{2}^{(k)} \in F_{1} \oplus \cdots \oplus F_{N}$ as in (e).

Take a bounded linear operator $T: \overline{\operatorname{span}}\left\{\widetilde{b}_{1}^{(k)}, \widetilde{b}_{2}^{(k)}\right\}_{k \geq 1} \rightarrow F_{N+1} \oplus \cdots \oplus$ $F_{N+4}$ which satisfies (9).

As in Section 3, start with a fixed $l \geq 1$ and put $\mathcal{M}_{i}=\left\{m: A_{N+i, m} \subset\right.$ $\left.A_{N+4, l}\right\}$ for $i=1,2,3$. Let $B \subset A_{N+4, l}$ be such that $\left|B \cap A_{N+1, r}\right|=1$ for all 
$r \in \mathcal{M}_{1}$. Thus, for every $s \in \mathcal{M}_{2}$, we have $B \cap A_{N+2, s} \in \mathcal{K}_{N+2, s}$ and by (4) we can find scalars $\left\{\beta_{k}\right\}_{k \in B \cap A_{N+2, s}}$ such that

$$
\left\|\sum_{k \in B \cap A_{N+2, s}} \beta_{k} f_{N+1, k}\right\| \geq 2^{s}, \quad\left\|\sum_{k \in B \cap A_{N+2, s}} \beta_{k} f_{N+2, k}\right\|=1 .
$$

Since $\left\{f_{N+2, k}\right\}_{k}$ is 1 -unconditional we get

$$
\left|\beta_{k}\right| \leq 1 \quad \text { for all } k \in B \cap A_{N+2, s} .
$$

Now pick the corresponding $t=t_{s} \in \mathcal{M}_{3}$ for which $B \cap A_{N+2, s} \subset A_{N+2, s} \subset$ $A_{N+3, t}$; also, by the initial choice of $B$, for every $k \in B \cap A_{N+2, s}$ there is a unique $r=r_{k} \in \mathcal{M}_{1}$ such that $k \in A_{N+1, r_{k}}$. Then

$$
\begin{aligned}
& \left\|\sum_{k \in B \cap A_{N+2, s}} \beta_{k} \widetilde{b}_{1}^{(k)}\right\| \\
& \leq\left\|\sum_{k \in B \cap A_{N+2, s}} 2^{-r_{k}} \beta_{k} p_{1}^{(k)}\right\|+2^{-t / 2}\left\|\sum_{k \in B \cap A_{N+2, s}} \beta_{k} f_{N+2, k}\right\| \\
& \quad+\left\|\sum_{k \in B \cap A_{N+2, s}} \beta_{k} f_{N+3, k}\right\|+\left\|\sum_{k \in B \cap A_{N+2, s}} \beta_{k} f_{N+4, k}\right\| \\
& \leq N \sum_{k \in B \cap A_{N+2, s}} 2^{-r_{k}}\left|\beta_{k}\right|+\left(1+4+4^{2}\right)\left\|\sum_{k \in B \cap A_{N+2, s}} \beta_{k} f_{N+2, k}\right\| \\
& \leq N+21 \leq C(N+4) .
\end{aligned}
$$

If we put this together with

$$
\begin{aligned}
& \left\|T\left(\sum_{k \in B \cap A_{N+2, s}} \beta_{k} \widetilde{b}_{1}^{(k)}\right)\right\| \\
& \quad \geq\left\|\sum_{k \in B \cap A_{N+2, s}} c_{k} 2^{-s / 2} \beta_{k} f_{N+1, k}\right\| \geq 2^{-s / 2} 2^{s} \min _{k \in B \cap A_{N+2, s}}\left|c_{k}\right|,
\end{aligned}
$$

we obtain a set $B^{\prime} \subset B$ such that $B^{\prime} \cap A_{N+3, t} \in \mathcal{K}_{3, t}$ for all $t \in \mathcal{M}_{3}$ and

$$
\left|c_{k}\right| \leq C(N+4)\|T\| \cdot 2^{-l / 2}
$$

for all $k \in B^{\prime}$ (here we used $\min \mathcal{M}_{2} \geq l$ ).

Now for every $t \in \mathcal{M}_{3}$ pick scalars $\left\{\beta_{k}\right\}_{k \in B^{\prime} \cap A_{N+3, t}}$ such that

$$
\left\|\sum_{k \in B^{\prime} \cap A_{N+3, t}} \beta_{k} f_{N+2, k}\right\| \geq 2^{t}, \quad\left\|\sum_{k \in B^{\prime} \cap A_{N+3, t}} \beta_{k} f_{N+3, k}\right\|=1 .
$$

Working with the series $\sum_{k \in B^{\prime} \cap A_{N+3, t}} \beta_{k} \widetilde{b}_{2}^{(k)}$ we can argue as before and get a set $B^{\prime \prime} \subset B^{\prime} \subset B$ with the property that $B^{\prime \prime} \cap A_{N+4, l} \in \mathcal{K}_{4, l}$ and

$$
\left|b_{k}\right| \leq C(N+4)\|T\| \cdot 2^{-l / 2} \quad \text { for all } k \in B^{\prime \prime} .
$$


Finally, let $\left\{\beta_{k}\right\}_{k \in B^{\prime \prime} \cap A_{N+4, l}}$ be scalars for which

$$
\left\|\sum_{k \in B^{\prime \prime} \cap A_{N+3, l}} \beta_{k} f_{N+3, k}\right\| \geq 2^{l}, \quad\left\|\sum_{k \in B^{\prime \prime} \cap A_{N+4, l}} \beta_{k} f_{N+4, k}\right\|=1 .
$$

Using the series $\sum_{k \in B^{\prime \prime} \cap A_{N+4, l}} \beta_{k}\left(\widetilde{b}_{1}^{(k)}-\widetilde{b}_{2}^{(k)}\right)$ we can find $k \in B^{\prime \prime} \subset A_{N+4, l}$ such that

$$
\left|a_{k}-b_{k}+c_{k}-d_{k}\right| \leq C(N+4)\|T\| \cdot 2^{-l},
$$

which combined with the previous estimates gives the conclusion of (e).

Assume now that we have managed to construct the desired vectors for some step $n \geq 2$ in our induction. In order to check the step $n+1$, we let $N_{n+1}=2 N_{n}+4$ and we start with (any) 1-unconditional decomposition $\left(F_{1} \oplus \cdots \oplus F_{N_{n}}\right) \oplus\left(F_{N_{n}+1} \oplus \cdots \oplus F_{N_{n}+4}\right) \oplus\left(F_{N_{n}+5} \oplus \cdots \oplus F_{2 N_{n}+4}\right) \subset Y$.

Similarly to Section 3 , for $k \geq 1$ let $v_{1}^{(k)}, \ldots, v_{n}^{(k)} \in F_{1} \oplus \cdots \oplus F_{N_{n}}$ be vectors obtained at the previous inductive step, renamed in order to simplify the notation. For $k \geq 1$ consider the vectors $x_{k}, y_{k} \in F_{N_{n}+1} \oplus \cdots \oplus F_{N_{n}+4}$ obtained at the inductive step $n=2$, defined similarly to (20). Again, for all $k \geq 1$ let $u_{1}^{(k)}, \ldots, u_{n}^{(k)}$ be vectors obtained at the previous step, this time constructed inside $F_{N_{n}+5} \oplus \cdots \oplus F_{2 N_{n}+4}$.

Now, for every $k \in A_{N_{n}+1, r} \cap A_{N_{n}+5, r^{\prime}}$ with $r=1,2, \ldots$ and $r^{\prime}=1,2, \ldots$, define inside $\left(F_{1} \oplus \cdots \oplus F_{N_{n}}\right) \oplus\left(F_{N_{n}+1} \oplus \cdots \oplus F_{N_{n}+4}\right) \oplus\left(F_{N_{n}+5} \oplus \cdots \oplus F_{N_{n+1}}\right)$ :

$$
\begin{array}{rlrl}
b_{1}^{(k)} & =2^{-r} v_{1}^{(k)}+\quad x_{k}, & \\
b_{2}^{(k)} & =2^{-r} v_{2}^{(k)}+ & +u_{1}^{(k)}, \\
& \vdots & & \\
b_{n}^{(k)} & =2^{-r} v_{n}^{(k)}+\quad u_{n-1}^{(k)}, \\
b_{n+1}^{(k)} & = & 2^{-r^{\prime}} y_{k}+u_{n}^{(k)},
\end{array}
$$

and then let $Y_{n+1}=\overline{\operatorname{span}}\left\{b_{1}^{(k)}, \ldots, b_{n+1}^{(k)}\right\}_{k \geq 1}$.

As in Section 3, at each step of the induction we are proving the following more precise statement for (c) and (d) (and (e)): for every $l \geq 1$ and every set $B_{l} \subset A_{N_{n}, l}$ (respectively $\left.B_{l} \subset A_{N_{n}+N, l}\right)$ such that $B_{l} \cap A_{1, m} \neq \emptyset$ whenever $A_{1, m} \subset A_{N_{n}, l}$ (respectively $B_{l} \cap A_{N+1, m} \neq \emptyset$ whenever $A_{N+1, m} \subset A_{N_{n}+N, l}$ ) there exists $k \in B_{l}$ such that the estimates of (c) and (d) (respectively (18)) are satisfied.

Let $T: Y_{n+1} \rightarrow Y_{n+1}$ be a bounded linear operator as in (c), that is, it satisfies $T\left(\operatorname{span}\left\{b_{1}^{(k)}, \ldots, b_{n+1}^{(k)}\right\}\right) \subset \operatorname{span}\left\{b_{1}^{(k)}, \ldots, b_{n+1}^{(k)}\right\}$ and $T_{\mid \operatorname{span}\left\{b_{1}^{(k)}, \ldots, b_{n+1}^{(k)}\right\}}$ $=\left[a_{i j}^{(k)}\right]_{1 \leq i, j \leq n+1}$ for all $k \geq 1$.

As mentioned above, fix $l \geq 1$ and start with a set $B_{l} \subset A_{N_{n+1}, l}$ such that $B_{l} \cap A_{1, m} \neq \emptyset$ whenever $A_{1, m} \subset A_{N_{n+1}, l}$. Put $\mathcal{M}_{1, l}=\left\{s: A_{N_{n}, s} \subset A_{N_{n+1}, l}\right\}$ and $\mathcal{M}_{2, l}=\left\{s: A_{N_{n}+4, s} \subset A_{N_{n+1}, l}\right\}$. 
We follow the same steps as in Section 3. The inductive hypothesis (d) applied to the vectors $v_{1}^{(k)}, \ldots, v_{n}^{(k)} \in F_{1} \oplus \cdots \oplus F_{N_{n}}($ with $k=1,2, \ldots$ ) and the operator $P_{F_{1} \oplus \cdots \oplus F_{N_{n}}} T_{\mid \overline{\operatorname{pan}}\left\{b_{1}^{(k)}, \ldots, b_{n}^{(k)}\right\}_{k>1}}: \overline{\operatorname{span}}\left\{b_{1}^{(k)}, \ldots, b_{n}^{(k)}\right\}_{k \geq 1} \rightarrow$ $F_{1} \oplus \cdots \oplus F_{N_{n}}$ implies the existence of indices $k_{s} \in B_{l} \cap A_{N_{n}, s}$, for all $s \in \mathcal{M}_{1, l}$, such that

$$
\begin{array}{r}
\max \left(\left\{\left|a_{i j}^{\left(k_{s}\right)}\right|: i \neq j, i, j \in\{1, \ldots, n\}\right\} \cup\left\{\left|a_{11}^{\left(k_{s}\right)}-a_{i i}^{\left(k_{s}\right)}\right|: 2 \leq i \leq n\right\}\right) \\
\leq 3 C\left(N_{n+1}\right)\|T\| \cdot 2^{-s / 2} 2^{r_{s}} .
\end{array}
$$

Here $r_{s}$ is the unique index with the property $A_{N_{n}, s} \subset A_{N_{n}+1, r_{s}}$. The extra constant $2^{r_{s}}$ in the estimate is a consequence of the definition (21) and this fact can be easily checked, first for the initial step of the induction and then for every step afterwards.

Without loss of generality we can assume that $s \geq 4 r_{s}$ for all $s \in \mathcal{M}_{1, l}$; this follows once we ensure that each member of the partition $\Delta_{N_{n}+1}$ is obtained by taking at least five successive members from the previous partition $\Delta_{N_{n}}$, which is allowed in the arguments from [14]. Since on the other hand we must have $r_{s} \geq l$, we can conclude that for every $s \in \mathcal{M}_{1, l}$,

$$
\begin{aligned}
\max \left(\left\{\left|a_{i j}^{\left(k_{s}\right)}\right|: i \neq j, i, j\right.\right. & \left.\in\{1, \ldots, n\}\} \cup\left\{\left|a_{11}^{\left(k_{s}\right)}-a_{i i}^{\left(k_{s}\right)}\right|: 2 \leq i \leq n\right\}\right) \\
& \leq 3 C\left(N_{n+1}\right)\|T\| \cdot 2^{-r_{s}} \leq 3 C\left(N_{n+1}\right)\|T\| \cdot 2^{-l} .
\end{aligned}
$$

Denote by $B_{l}^{\prime} \subset B$ the set formed by such indices $k_{s}$. Using now (e) for the initial inductive step, for the vectors $x_{k}, y_{k} \in F_{N_{n}+1} \oplus \cdots \oplus F_{N_{n}+4}$

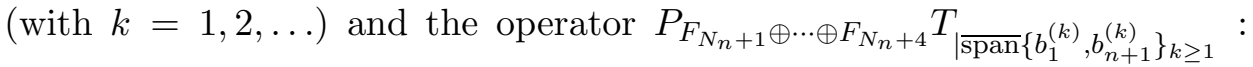
$\overline{\operatorname{span}}\left\{b_{1}^{(k)}, b_{n+1}^{(k)}\right\}_{k \geq 1} \rightarrow F_{N_{n}+1} \oplus \cdots \oplus F_{N_{n}+4}$, we find that for every $s \in \mathcal{M}_{2, l}$ there is $k_{s} \in B_{l}^{\prime} \cap A_{N_{n}+4, s}$ such that

$$
\begin{aligned}
\max \left\{\left|a_{1, n+1}^{\left(k_{s}\right)}\right|,\left|a_{n+1,1}^{\left(k_{s}\right)}\right|,\right. & \left.\left|a_{11}^{\left(k_{s}\right)}-a_{n+1, n+1}^{\left(k_{s}\right)}\right|\right\} \\
& \leq 3 C\left(N_{n+1}\right)\|T\| \cdot 2^{-s / 2} 2^{r_{s}^{\prime}} \\
& \leq 3 C\left(N_{n+1}\right)\|T\| \cdot 2^{-r_{s}^{\prime}} \leq 3 C\left(N_{n+1}\right)\|T\| \cdot 2^{-l} .
\end{aligned}
$$

As before, for every $s \in \mathcal{M}_{2, l}, r_{s}^{\prime}$ denotes the unique index such that $A_{N_{n}+4, s} \subset A_{N_{n}+5, r_{s}}$; in addition, we assumed without loss of generality that in such a case we have $s \geq 4 r_{s}^{\prime}$.

Let $B_{l}^{\prime \prime} \subset B_{l}^{\prime}$ be the collection of all such indices $k_{s}$ as above. We would like now to apply the inductive hypothesis (e) to the vectors $u_{1}^{(k)}, \ldots, u_{n}^{(k)} \in$ $F_{N_{n}+5} \oplus \cdots \oplus F_{N_{n+1}}(k \geq 1)$. Notice that for every $k \in A_{N_{n}+5, r^{\prime}}\left(\right.$ with $r^{\prime}=$ $1,2, \ldots)$ the vectors $b_{2}^{(k)}, \ldots, b_{n+1}^{(k)}$ are suitable perturbations of $u_{1}^{(k)}, \ldots, u_{n}^{(k)}$, of the type considered in (19); this is a consequence of (21) and the fact that whenever $k \in A_{N_{n}+1, r} \cap A_{N_{n}+5, r^{\prime}}$ we must necessarily have $r \geq r^{\prime}$. 
Thus (e) applied to the operator $P_{F_{N_{n}+5} \oplus \cdots \oplus F_{N_{n+1}}} T_{\mid \overline{\operatorname{span}}\left\{b_{2}^{(k)}, \ldots, b_{n+1}^{(k)}\right\}_{k \geq 1}}$ : $\overline{\operatorname{span}}\left\{b_{2}^{(k)}, \ldots, b_{n+1}^{(k)}\right\}_{k \geq 1} \rightarrow F_{N_{n}+5} \oplus \cdots \oplus F_{N_{n+1}}$ yields $k \in B_{l}^{\prime \prime}$ such that $\max \left\{\left|a_{i j}^{(k)}\right|: i \neq j, i, j \in\{2, \ldots, n+1\}\right\} \leq 3 C\left(N_{n+1}\right)\|T\| \cdot 2^{-l / 2}$.

This gives the conclusion of (c) for such $k \in A_{N_{n+1}, l}$.

For the statements (d) and (e), it is not hard to see that by following the same line of argument as before one can obtain the desired conclusions.

5. Proof of main result. We will now present the proof of Theorem 1.1 .

Let $Y \subset X$ be a subspace with an unconditional basis and assume that it does not contain a subspace isomorphic to $\ell_{2}$. By renorming if necessary, we may assume that $Y$ has a 1-unconditional basis $\left\{e_{i}\right\}_{i}$. Consider an arbitrary partition of the basis $\left\{e_{i}\right\}_{i}$ into infinite subsets $\left\{e_{i}\right\}_{i \in I_{1}},\left\{e_{i}\right\}_{i \in I_{2}}, \ldots$

In the case that there is $k_{0} \geq 1$ such that $E_{0}:=\overline{\operatorname{span}}\left\{e_{i}\right\}_{i \in I_{k_{0}}}$ has finite cotype, we can proceed with the construction from Sections 3 and 4 and obtain, for every $n \geq 2$, a subspace $Y_{n} \subset E_{0}$ which admits a 1-unconditional $n$-uniform decomposition and, at the same time, $\mathcal{U}_{n-1}\left(Y_{n}\right)=\infty$. In fact, by partitioning $I_{k_{0}}$ one more time, we can ensure that each of the spaces $\left\{Y_{n}\right\}_{n \geq 2}$ is supported on disjoint elements of $\left\{e_{i}\right\}_{i \in I_{k_{0}}}$. Thus we can define $Z=\sum_{n \geq 2} \oplus Y_{n} \subset E_{0}$ and obtain a space with a 1-unconditional finitedimensional decomposition (as in the case of each $Y_{n}$, this is a consequence of Remark 2.3). As well, for every $n \geq 2, Y_{n}$ is 1-complemented in $Z$ and therefore we must have $\mathcal{U}_{n-1}(Z)=\infty$.

Suppose now that $E_{k}:=\overline{\operatorname{span}}\left\{e_{i}\right\}_{i \in I_{k}}$ does not have finite cotype, for $k=1,2, \ldots$, which is equivalent to the fact that each of these spaces contains $\ell_{\infty}^{n}$ 's uniformly. For every $k \geq 1$, let $F_{k} \subset E_{k}$ be a subspace such that $\operatorname{dim} F_{k}=k$ and $d\left(F_{k}, l_{\infty}^{k}\right) \leq 2$, where $d$ stands for the Banach-Mazur distance. Since, by a classical result of Figiel and Johnson [5] from local theory, a random [ $k / 2]$-dimensional subspace of $\ell_{\infty}^{k}$ has the Gordon-Lewis constant of maximal order, for every $k \geq 1$ there exists a [k/2]-dimensional subspace $Z_{k}$ of $F_{k}$ satisfying $\operatorname{GL}\left(Z_{k}\right) \geq c \sqrt{k}$, with $c$ an absolute constant. By [2, Proposition 1.3], for $n=1,2, \ldots$ we have

$$
\sqrt{n} \mathcal{U}_{n}\left(Z_{k}\right) \geq \mathrm{GL}\left(Z_{k}\right) \geq c \sqrt{k} \quad \text { for all } k \geq 1 .
$$

If we now let $Z=\sum_{k \geq 1} \oplus Z_{k} \subset Y$, then clearly $Z$ has a 1-unconditional finite-dimensional decomposition. On the other hand, for every $k \geq 1, Z_{k}$ is 1 -complemented in $Z$, while $\sup _{k} \mathcal{U}_{n}\left(Z_{k}\right)=\infty$ for $n=1,2, \ldots$ This shows that $\mathcal{U}_{n}(Z)=\infty$ for all $n \geq 1$.

Acknowledgements. This research was supported in part by NSERC Grant 312594-05. Part of this work was finished when the author attended 
the "Workshop in analysis and probability" of Texas A\&M University in July 2006, whose support is greatly appreciated.

\section{References}

[1] R. Anisca, Unconditional decompositions in subspaces of $l_{2}(X)$, Positivity 8 (2004), 423-441.

[2] A. Borzyszkowski, Unconditional decomposition and local unconditional structures in some subspaces of $L_{p}, 1 \leq p<2$, Studia Math. 76 (1983), 267-278.

[3] P. G. Casazza and N. J. Kalton, Unconditional bases and unconditional finitedimensional decompositions in Banach spaces, Israel J. Math. 95 (1996), 349-373.

[4] V. Ferenczi and C. Rosendal, On the number of non-isomorphic subspaces of a Banach space, Studia Math. 168 (2005), 203-216.

[5] T. Figiel and W. B. Johnson, Large subspaces of $l_{\infty}^{n}$ and estimates of the GordonLewis constant, Israel J. Math. 37 (1980), 92-112.

[6] W. T. Gowers, A new dichotomy for Banach spaces, Geom. Funct. Anal. 6 (1996), 1083-1093.

[7] W. B. Johnson, J. Lindenstrauss and G. Schechtman, On the relation between several notions of unconditional structure, Israel J. Math. 37 (1980), 120-129.

[8] T. Ketonen, On unconditionality in $L_{p}$ spaces, Ann. Acad. Sci. Fenn. Ser. A I Math. Diss. 35 (1983).

[9] R. Komorowski and N. Tomczak-Jaegermann, Banach spaces without local unconditional structure, Israel J. Math. 89 (1995), 205-226.

[10] - - -, Eratum to: Banach spaces without local unconditional structure, ibid. 105 (1998), 85-92.

[11] - - - Subspaces of $l_{2}(X)$ and $\operatorname{Rad}(X)$ without local unconditional structure, Studia Math. 149 (2002), 1-21.

[12] J. Lindenstrauss and L. Tzafriri, Classical Banach Spaces I. Sequence Spaces, Springer, Berlin, 1977.

[13] N. Tomczak-Jaegermann, Banach-Mazur Distances and Finite-Dimensional Operator Ideals, Pitman Monogr. Surveys Pure Appl. Math. 38, Longman Sci. Tech., Harlow, 1989.

[14] - A solution of the homogeneous Banach space problem, in: Canadian Mathematical Society 1945-95 Anniversary Volume, Canad. Math. Soc., Ottawa, 1996, Vol. 3, 267-286.

Department of Mathematical Sciences

Lakehead University

Thunder Bay, ON, Canada P7B 5E1

E-mail: ranisca@lakeheadu.ca

Received October 18, 2006

Revised version March 29, 2007 